\title{
Experimental Investigation of High Pulse Power Microwave Resistive Sensor with Flat Frequency Response
}

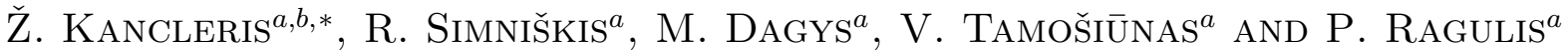 \\ ${ }^{a}$ Semiconductor Physics Institute, A. Goštauto 11, LT-01108 Vilnius, Lithuania \\ ${ }^{b}$ Faculty of Physics, Vilnius University \\ Saulètekio al. 9, LT-10222 Vilnius, Lithuania
}

\begin{abstract}
Two types of resistive sensors with flat frequency response for microwave pulse power measurements in X-band rectangular waveguide WR-90 up to $100 \mathrm{~kW}$ were developed and experimentally investigated. The first type of the sensors demonstrates higher sensitivity and larger output signal, while the second one exhibits better linearity of the output signal. The experimental investigations revealed the sensitivity variation within $\pm 10 \%$ in 8.2-12.2 GHz frequency band.
\end{abstract}

PACS numbers: 07.57.Kp, 72.20.Ht, 85.30.De

\section{Introduction}

High power microwave (HPM) pulse sensors are important in HPM pulse diagnostics, research and development. A resistive sensor (RS) is one of the promising devices for HPM pulse applications, when the fast response, stability and resistibility to overload and breakdown are of the most importance [1]. Several types of the RS for different HPM pulse power level and duration were developed. One of the most successful solutions is the RS with a sensing element (SE) mounted between a stretched metal diaphragm and a wide wall of the rectangular waveguide [2]. The main drawback of the diaphragm type RS up to now was a large variation of the sensitivity in a waveguide's frequency range. In our previous paper [3], the diaphragm type RS with flat frequency response was engineered using finite-difference time domain (FDTD) method. Here the experimental investigations of the optimised RS with diaphragm are presented. Although the measured characteristics of the diaphragm type RS, in general, confirm the predicted one [3], it was found that its output characteristic is sufficiently nonlinear at HPM region. Therefore, we also investigated the alternative sensor with flat frequency response composed of two SE mounted on a wide wall of the waveguide. It demonstrates lower sensitivity in comparison with the diaphragm type RS but better linearity in the HPM region.

\section{Investigated sensors}

Cross-sectional views of the investigated sensors are shown in Fig. 1. The sketch of the diaphragm type RS is shown in (a) and (b), whereas in (c) and (d) the RS with two SE is displayed. As seen from Fig. 1, the SE of the diaphragm RS is grounded by metal diaphragm 2 , while in the case of two SE, one of them is mounted on the hot contact, while the other one is grounded. Upper contacts of sensing elements are shorted with thin metal foil. The electric field of the microwave pulse heats current carriers in the SE and its resistance increases. Applying constant current feed to the sensor, the increase of its resistance causes the voltage pulse signal in the output.

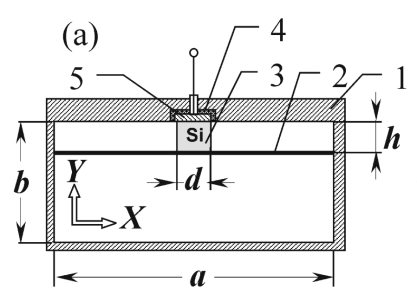

(c)

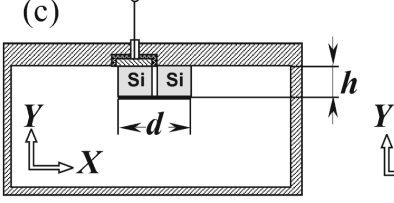

(b)

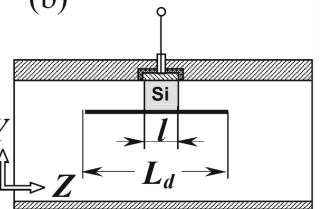

(d)

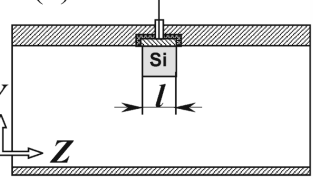

Fig. 1. Sensors for high power pulse measurement in the waveguide: (a), (b) the RS with diaphragm, (c) and (d) the RS with two SE. (a) and (c) are the frontal, (b) and (d) side cross-sectional views. 1 is a rectangular waveguide, 2 - metallic diaphragm, 3 - sensing element with ohmic contacts, 4 - insulating washer, 5 - isolated contact. 


\section{Sensitivity}

The sensitivity of the RS is defined as:

$$
\zeta=\frac{\Delta R}{R} \frac{1}{P}=\frac{\beta^{*}\left\langle E_{\mathrm{m}}\right\rangle^{2}}{P},
$$

where $\Delta R / R$ is a relative resistance change, $P$ is a power transmitted through the waveguide, $\beta^{*}$ is an effective warm-electron coefficient in RF electric field and $\left\langle E_{\mathrm{m}}\right\rangle$ is an averaged amplitude of RF electric field in the SE. Considering dependence of $\zeta$ on frequency it was established [3] that even for the same power level transmitted through the waveguide, electric field strength in it decreases with frequency because of waveguide dispersion. For X-band RS the waveguide dispersion is the only factor influencing the sensitivity of the RS and causing its decrease with frequency. Therefore the objective of the FDTD simulation was to find electrophysical parameters of the RS providing the increase of $\left\langle E_{\mathrm{m}}\right\rangle$ with frequency and in this way getting the flat frequency response of the RS (1). We performed such a simulation and the parameters of the optimal diaphragm type RS [3] and the optimal RS with two SE are collected in Table. Calculated dependences of $\zeta$ on frequency are shown in Fig. 2. It is worth to mention that $\left\langle E_{\mathrm{m}}\right\rangle$ in the diaphragm type RS is roughly equal to the amplitude of the electric field in the centre of the empty waveguide whereas in the case of the RS with two SE, $\left\langle E_{\mathrm{m}}\right\rangle$ is about four times smaller.
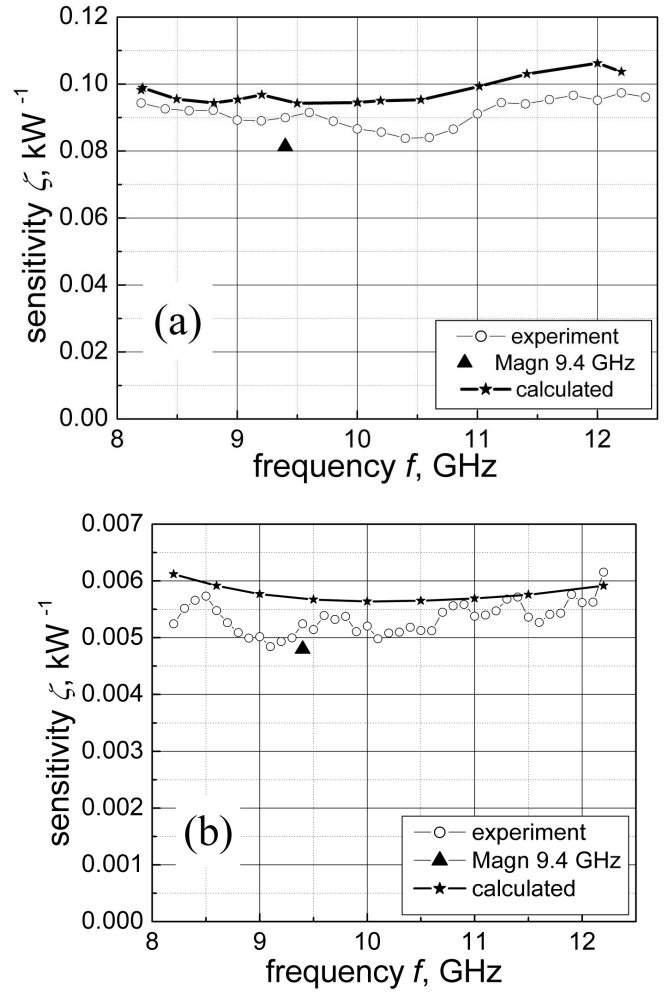

Fig. 2. Frequency response of the optimised diaphragm type RS (a), and the optimised RS with two $\mathrm{SE}$ (b). $\Delta$ high power measurements at $9.4 \mathrm{GHz}$.
TABLE

Main specifications of the optimized sensors.

\begin{tabular}{l|c|c|c|c|c}
\hline \hline $\begin{array}{c}\text { Sensor } \\
\text { type }\end{array}$ & $\begin{array}{c}h \times d \times l \\
{\left[\mathrm{~mm}^{3}\right]}\end{array}$ & $\begin{array}{c}L \\
{[\mathrm{~mm}]}\end{array}$ & $\begin{array}{c}\rho \\
{[\Omega \mathrm{cm}]}\end{array}$ & $\begin{array}{c}\zeta \\
{\left[\mathrm{kW}^{-1}\right]}\end{array}$ & $\begin{array}{c}\Delta \zeta \\
{[\%]}\end{array}$ \\
\hline diaphragm & $1 \times 0.75 \times 0.75$ & 20 & 50 & 0.09 & \pm 10 \\
two SE & $1 \times 3 \times 1.5$ & - & 30 & 0.0055 & \pm 10
\end{tabular}

The prototypes of the optimal RS were fabricated and the measurements of frequency response were performed using $100 \mathrm{~W}$ TWT amplifier and reference average power sensor Rohde/Schwarz NRV-Z54. The results of measurements are also presented in Fig. 2. It is seen that the reasonable agreement between calculated and measured values of $\zeta$ has been obtained.

\section{Output signal}

The output signal dependences on pulse power have been measured up to $100 \mathrm{~kW}$ at $9.4 \mathrm{GHz}$ and the dependences of $\Delta R / R$ on $P$ have been determined for both types of the RS. Experimental results are presented in Fig. 3 by points. Solid lines in the figure show fitting of the measured dependence by the second order polynomial
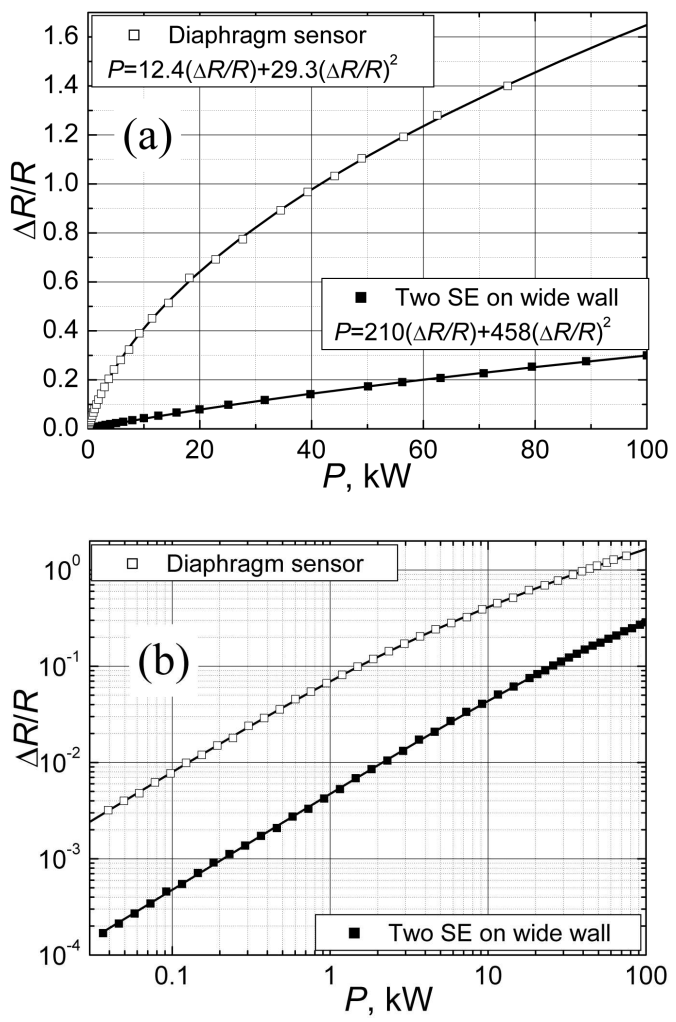

Fig. 3. Dependences of the relative resistance change on pulse power for the diaphragm type RS (open squares) and the RS with two SE in the linear (a) and logarithmic (b) scales. 


$$
P=A(\Delta R / R)+B(\Delta R / R)^{2},
$$

where coefficients $A=1 / \zeta$ and $B$ are determined by fitting experimentally measured dependence with (2). The values of the coefficients $A$ and $B$ for particular sensor are shown in the inset in Fig. 3. The values of sensitivity $\zeta$ determined from high power test are shown in Fig. 2 by solid triangles. As one can see from Fig. 3, the RS with two SE demonstrates more linear characteristic in the HPM region in comparison with the diaphragm type RS.

\section{Conclusions}

The optimal parameters of two types of the RS were found and the optimal RSs were manufactured and exper- imentally investigated. Both demonstrate roughly $\pm 10 \%$ sensitivity variation within waveguide's frequency range. The RS with two SE exhibits lower sensitivity but better linearity in the HPM region in comparison with the diaphragm type RS.

\section{References}

[1] M. Dagys, Ž. Kancleris, R. Simniškis, E. Schamiloglu, F.J. Agee, IEEE Antennas Propag. Mag. 43, 64 (2001).

[2] M. Dagys, Ž. Kancleris, V. Orševskis, R. Simniškis, Electron. Lett. 31, 1355 (1995).

[3] Ž. Kancleris, V. Tamošiūnas, M. Dagys, R. Simniškis, F.J. Agee, IEEE Microw. Wirel. Comp. Lett. 16, 422 (2006). 